\title{
Increased output in micro production by tolerance field widening and synchronisation
}

\author{
Ann-Kathrin Onken ${ }^{1,2^{*}}$, Philipp Wilhelmi ${ }^{1,2}$, Kirsten Tracht ${ }^{1,2,3}$, and Bernd Kuhfuss ${ }^{1,2,3}$ \\ ${ }^{1}$ University of Bremen, Bibliothekstr. 1, 28359 Bremen, Germany \\ ${ }^{2}$ Bremen Institute for Mechanical Engineering, Badgasteiner Str. 1, 28359 Bremen, Germany \\ ${ }^{3}$ MAPEX Center for Materials and Processing, Am Fallturm 1, 28359 Bremen, Germany
}

\begin{abstract}
The manufacturing of linked micro parts simplifies the handling and facilitates a consideration of trends due to the maintaining of the manufacturing order. These known changes of geometrical characteristics of parts are the basis for softening tolerances. The basic idea of the tolerance field widening is familiar with selective assembly. Instead of single parts, trend-based sections are used for increasing the number of assemblies under consideration of the clearance. The outcome of the assembly of the identified trend-based sections is influenced by the occurring trends and the changes of trends. Therefore, based on simulated data the effects of occurring trends are investigated. For sorting and matching the identified trend-based sections in high quantities, a case specific pre-assembly in the synchronisation point is required. Possible synchronisation scenarios as well as a synchronisation station for the synchronisation of two types of linked parts are presented. The results for the influence of trends show that the tolerance field widening increases the outcome especially for intersecting and opposing trends.
\end{abstract}

Keywords: Assembly, Handling, Tolerances

\section{Introduction}

Mass production of micro parts at high output rates is still challenging in industrial applications. On the one hand, the occurrence of size effects [1] and sensitive parts [2] rises the complexity of handling. On the other hand, in most cases, small tolerances [3] are required for assembly. The high demand for micro parts, as well as the resulting demand for economic production and handling processes, [4] shows the necessity for new methodologies in micro production. From the technological point of view, the output rates can be increased by simplifying and speeding up handling operations. The method of handling micro parts as linked parts [5] avoids micro-specific challenges directly by concept. The design of linked micro parts depends on the linkage of the micro parts and is case specific, with a possible differentiation between the three basic types ladder type, line type and comb [2].

The production with linked parts is investigated at the University of Bremen for two exemplary production processes within the collaborative research centre 747 . The manufacturing process of ladder linked parts is shown in Figure 1. The parts are produced on base of sheet metal by stretch drawing. The manufacturing process of the line linked parts is based on wire material. In the first step, preforms are manufactured by laser rod melting and in the second step, the preforms are processed by rotary swaging, see Figure 2.

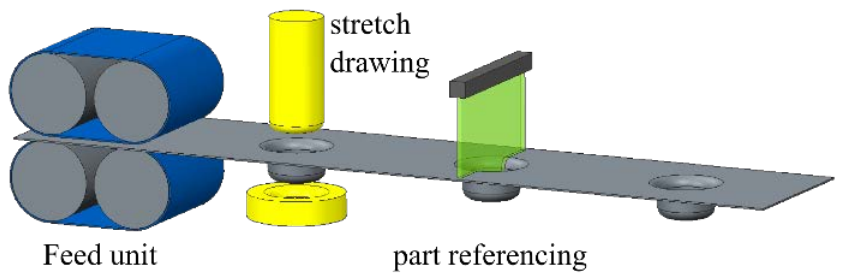

Fig. 1. Manufacturing process of ladder linked parts

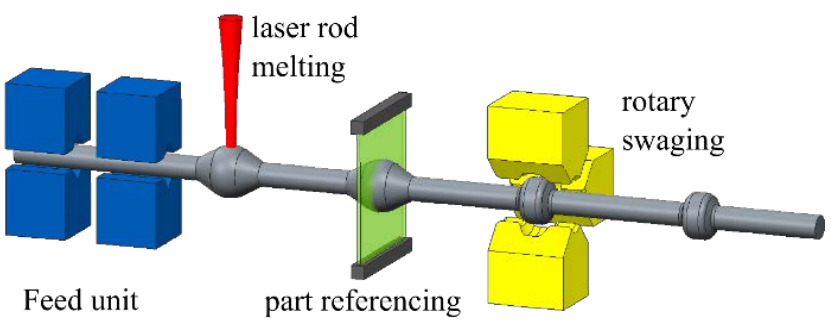

Fig. 2. Manufacturing process of line linked parts

The interconnection of the parts decreases the complexity of handling [5] and maintains the manufacturing order. The sustainment of the manufacturing order enables an identification and consideration of trends. The basic idea behind this, is familiar with selective assembly. Until now, methodologies of selective assembly are mainly applied for processes that cannot guarantee the production of parts within required tolerances. Kumar and Kannan investigated genetic algorithms for obtaining optimal

\footnotetext{
* Corresponding author: onken@bime.de
} 
manufacturing tolerances [6]. Studies for applying selective assembly and adaptive production are described by Lanza [7] or Colledani [8]. Within these approaches, the tolerances are used for direct consideration in further manufacturing processes. Raj et al. [9] investigated the selective assembly of batches, by using a non-dominated sorting genetic algorithm. This approach is only applicable for small or medium sized batches.

The fact that linked parts are arranged according to the production time enables an extension of the application spectrum of selective assembly by tolerance field widening to established processes like stretch drawing. These processes are generally able to produce parts within required variances. In this case, an increased output can be achieved by softening tolerances. In case of defects in micro parts like valves, the whole product is interchanged. For this reason, the tolerances could be defined for every single assembly. The basic idea of the tolerance field widening is illustrated in Figure 3. It is assumed that the geometrical features of the produced parts show a mean variance, which is small compared to the tolerances, but that the mean value may show trends. These trends can be caused for example by tool wear. In [10], the concept for tolerance field widening for the production of linked parts in three steps is presented. Firstly, trends of the first batches of the process chains are considered and the linked parts are divided into clusters. Then clusters from linked parts of both types are matched. Finally, the process parameters are adapted, in order to maximize the number of successfully matched parts, for the following produced parts. Trends are occurring, especially in forming processes, due to e.g. wear. Therefore, this approach is specifically useful in this case.

Production of the first batch

Tolerance field widening

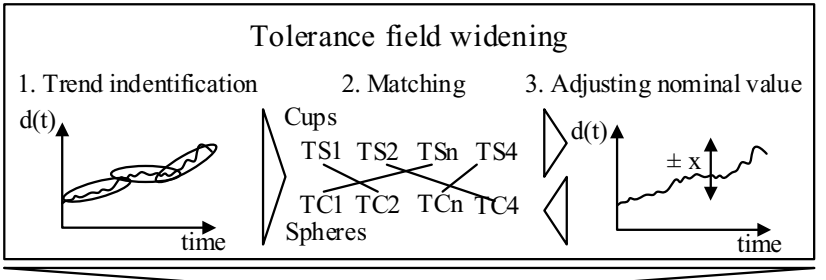

Pre-assembly of further batches in synchronisation point

$\mathrm{d}=$ Diameter $\quad \mathrm{TSn}=$ Trend spheres $\quad \mathrm{TCn}=$ Trends Cups $\quad \mathrm{x}=$ Adjustment

Fig. 3. Concept of tolerance field widening in linked parts production

For assuring a cost effective micro mass production, technological aspects, as well as the softening of tolerances, has to be considered. These two areas of research interdepend, especially in the synchronisation station, where the produced parts of the different process chains are synchronised. Possible scenarios for synchronisation as well as a design of a synchronisation station based on the example of cups and spheres are introduced. Trends that occur during production processes, influence the geometrical characteristics of micro parts. Different distribution will lead to varying numbers and sizes of sections. The number of sections is limited due to the objective of short cycle times in the synchronisation station. For identification of a trendsspecific number of production sections, the influence of trends on the number of pre-assemblies in interdependence with the number of sections, is investigated based on simulation studies.

\section{Appearance of trends}

Changing states of components over time, are already addressed in research. The first field of research is within through life engineering services and the second one is within tools. In both fields, occurring wear and timing of tool or part changes are predicted. Up to sixteen wear mechanisms are considered in literature, that are caused by mechanical action, chemical action, or thermal influences [11]. Hsu et al. identified 32 different parameters that have been used for describing and investigating wear [12]. Looking at through life engineering services, the prediction of wear is based on the consideration of various parameters and environmental influences. The Weibull curve [13] is often used within prediction models and approaches. In the research field of manufacturing processes, the emphasis is the endurance of tools. Areas of interest are for example, the prediction of wear and end of lifetime. Belfiore et al. introduces an approach for wear and fatigue prediction in metal forming, by multilayer neural networks [14]. Furthermore, the influence of surface properties on wear is investigated. Yilkiran et al. present the influence of oxidised tool surfaces [15]. The mechanisms are of further interest. Liu et al differentiate wear patterns and mechanisms of cutting tools in milling [16]. The delineation of wear is also described in literature. Ber and Kaldor describe the course of wear in cutting processes [17]. Boher et al. investigated the influence of wear behaviour on the radius portion and identified that the surface degradation is caused by adhesion and ploughing [18]. The necessity of lubricant on wear in combined blanking and deep- drawing process of micro parts, due to the occurrence of flakes, was investigated by Flosky and Vollertsen [19]. In general, the influence of tool wear on the geometrical changes of parts, over the manufacturing time, is not described. Weikert et al. identified a trend in the measured diameter of produced micro parts that were investigated for statistical studies [20]. Changes within the trend were not described since only a minor number of parts were considered. Due to the missing information, data with trend changes were simulated. For identifying in which way trends and their changes influence the number of pre-assemblies and the number of sections, especially if they are limited, the scenarios for identifying boundaries are simulated.

\section{Synchronisation of micro parts}

Synchronisation in the context of different process chains is usually understood as an adaption of the cycle rates, in order to have the same output. For the following considerations the term of synchronisation is defined different and respectively extended to the production of 
linked parts, in combination with tolerance field widening. It is aspired to maintain the interconnection, not only during the single process chains, but also for the part supply in assembly. For that purpose, at least two parts, for example type A sphere and type B cup, need to be provided at a certain time to a certain point (synchronisation point) and ideally with a defined orientation. When applying the tolerance field widening to the linked parts production, an additional condition counts for the synchronisation. In order to produce a functional assembly, it is not longer sufficient that a random part from type $\mathrm{A}$ is synchronised with a random part from type B. A part from a specific cluster of the linked parts of type A must be synchronised with a part from a specific cluster of the linked parts of type B. The operation performed in the synchronisation point must not necessarily be an assembly, but can also be the creation of a new type of linked parts, containing pairs of synchronised parts. In case of creating a new type of linked parts there are theoretically the three following options.

- The linked parts of both types are joined directly without separating them before

- The linked parts of both types are separated and a new linkage is created

- The linked parts of one type are separated and joined to the linked parts of the other type

For a direct joining as proposed, in case one the distances between the parts in both linked parts must be exactly the same. Furthermore, the separation of such linked parts would be more difficult compared to the other cases. For the creation of a completely new type of linked parts, the number of necessary operations is much higher. Firstly, both linked parts must be separated and then joined again. By these considerations it results, that in most cases the third option is the most convenient. For the exemplary process chains described before there are the two options illustrated in Figure 4.

a)

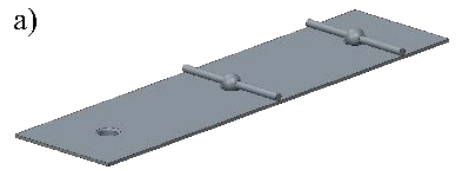

Fig.4. Synchronised linked parts

As they are produced of thin metal foil, the cups in the ladder linked parts are much more sensitive against mechanical damage. Consequently, for the further handling, it is easier to maintain the ladder linked parts and separate the line linked parts (Figure 4a). To investigate the synchronisation, a synchronisation station has been designed for that case. It applies all the developed technologies from the single process chains, such as feed units [21] and high speed referencing system, which enable a fast and accurate positioning within the synchronisation point [22]. The central module of the synchronisation station is illustrated in Figure 5. The separation of the line linked parts is realised by mechanical shearing and the joining of the linked parts is performed via capacitor discharge welding. Pneumatic cylinders realise the mechanical actuation.

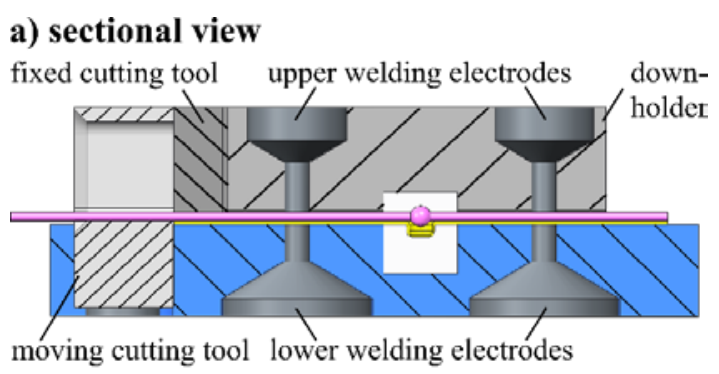

\section{b) top view}

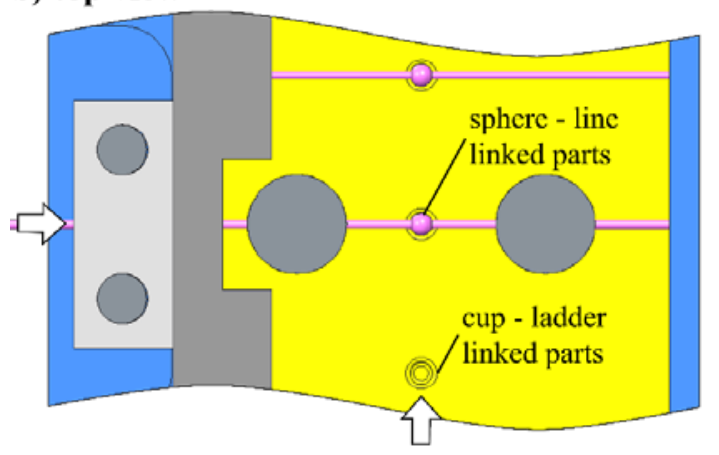

Fig.5. Central synchronisation module

The synchronisation module is designed for assembly of more than 200 parts per minute. While implementing the results of the tolerance field widening on the synchronisation station, every change of linked parts and therefore the total number of sections that results in the identified clusters causes an interruption of the assembly process. For assuring high quantities, the number of sections should be as low as possible. For this reason, the influence of trends on the number of sections is investigated in the following.

\section{Simulation setup}

Depending on the process chains for the produced linked parts, different trends may be identified in the part dimensions. Based on the example of cups and spheres, four different trend scenarios and three different intensities of trends are assumed, which result in twelve data sets. For each of the twelve data sets, two thousand data-points are simulated. Variances are considered by adding a random value between $-0,0005 \mu \mathrm{m}$ and $+0,0005 \mu \mathrm{m}$. The aspired fitting tolerance is assumed to be between $0 \mu \mathrm{m}$ and $10 \mu \mathrm{m}$. This small clearance assures that not all parts could be matched, for showing the influence of the trends on the number of sections and on the number of assemblies.

In Figure 6 the four different trend scenarios are shown. In all twelve data sets the nominal value of the diameter of the spheres starts at $908.2 \mu \mathrm{m}$ and the diameter of the cups at $890.8 \mu \mathrm{m}$. Within the scenarios 1 and 2, changing linear trends and in the scenarios 3 and 4 the combination of linear and quadratic trends are simulated.

The three datasets of a scenario have the same direction of trends with varying intensities as listed in Table 1 . The 
Table 1. Intensity of trends in $\mu \mathrm{m}$.

\begin{tabular}{|c|c|c|c|c|c|c|c|c|c|c|c|c|}
\hline $\mathrm{T}$ & $\mathrm{D} 1.1$ & $\mathrm{D} 1.2$ & $\mathrm{D} 1.3$ & $\mathrm{D} 2.1$ & $\mathrm{D} 2.2$ & $\mathrm{D} 2.3$ & $\mathrm{D} 3.1$ & $\mathrm{D} 3.2$ & $\mathrm{D} 3.3$ & $\mathrm{D} 4.1$ & $\mathrm{D} 4.2$ & $\mathrm{D} 4.3$ \\
\hline C 1 & 0.001 & 0.0001 & 0.0005 & -0.001 & -0.0001 & -0.0005 & 0.001 & 0.0001 & 0.0005 & -0.001 & -0.0001 & -0.0005 \\
\hline C 2 & 0.0011 & 0.00011 & 0.00055 & -0.0011 & -0.00011 & -0.00055 & 0.0011 & 0.00011 & 0.00055 & -0.0011 & -0.00011 & -0.00055 \\
\hline C 3 & 0.0013 & 0.00013 & 0.00066 & -0.0013 & -0.00013 & -0.00066 & 0.0013 & 0.00013 & 0.00066 & -0.0013 & -0.00013 & -0.00066 \\
\hline C 4 & 0.00135 & 0.000135 & 0.00074 & -0.00135 & -0.000135 & -0.00074 & 0.1 & 0.2 & 0.2 & -0.1 & -0.2 & -0.2 \\
\hline C 5 & 0.00145 & 0.000145 & 0.0008 & -0.00145 & -0.000145 & -0.0008 & 0.00145 & 0.000145 & 0.0008 & -0.00145 & -0.000145 & -0.0008 \\
\hline C 6 & 0.0015 & 0.00015 & 0.001 & -0.0015 & -0.00015 & -0.001 & 0.0015 & 0.00015 & 0.001 & -0.0015 & -0.00015 & -0.001 \\
\hline C 7 & 0.0016 & 0.00016 & 0.0015 & -0.0016 & -0.00016 & -0.0015 & 0.12 & 0.3 & 0.4 & -0.12 & -0.3 & -0.4 \\
\hline C 8 & 0.0017 & 0.00017 & 0.0017 & 0.0017 & -0.00017 & 0.0017 & 0.15 & 0.4 & 0.7 & -0.15 & -0.4 & -0.7 \\
\hline S 1 & 0.0009 & 0.0009 & 0.0003 & 0.0009 & 0.0009 & 0.0003 & 0.0009 & 0.0009 & 0.0003 & 0.0009 & 0.0009 & 0.0003 \\
\hline S 2 & 0.001 & 0.001 & 0.00035 & 0.001 & 0.001 & 0.00035 & 0.001 & 0.001 & 0.00035 & 0.001 & 0.001 & 0.00035 \\
\hline S 3 & 0.0012 & 0.0012 & 0.0005 & 0.0012 & 0.0012 & 0.0005 & 0.0012 & 0.0012 & 0.0005 & 0.0012 & 0.0012 & 0.0005 \\
\hline S 4 & 0.00129 & 0.00129 & 0.00129 & 0.00129 & 0.00129 & 0.00129 & 0.00129 & 0.00129 & 0.00129 & 0.00129 & 0.00129 & 0.00129 \\
\hline S 5 & 0.00133 & 0.00133 & 0.00133 & 0.00133 & 0.00133 & 0.00133 & 0.00133 & 0.00133 & 0.00133 & 0.00133 & 0.00133 & 0.00133 \\
\hline S 6 & 0.0015 & 0.0015 & 0.0015 & 0.0015 & 0.0015 & 0.0015 & 0.0015 & 0.0015 & 0.0015 & 0.0015 & 0.0015 & 0.0015 \\
\hline S 7 & 0.00155 & 0.00155 & 0.00155 & 0.00155 & 0.00155 & 0.00155 & 0.00155 & 0.00155 & 0.00155 & 0.00155 & 0.00155 & 0.00155 \\
\hline S 8 & 0.0016 & 0.0016 & 0.0016 & 0.0016 & 0.0016 & 0.0016 & 0.0016 & 0.0016 & 0.0016 & 0.0016 & 0.0016 & 0.0016 \\
\hline
\end{tabular}

first dataset of every scenario shows similar trends with lower trend variations.

Within the second dataset, the trend of one kind of parts is distinguished by higher trend intensities and higher changes. For the third data set of the scenarios, the diameters of both kind of parts has intensive trends and intensive changes. The trends ( $\mathrm{T}$ ) in $\mu \mathrm{m}$ of the data sets (D) are changing every 250 parts of cups (C) and spheres (S). The table elements with grey background do have a quadratic trend. These trends were considered using the following equation:

$$
d(t)=d(t-1)+T * x^{2} .
$$

$\mathrm{X}$ starts at 0.01 and increases in 0.01 steps over the 250 iterations to 0.250 . Due to the fact, that in accordance to minimal cycle times only a limited number of sections is contemplated a limited number of sections is considered. For the investigation of trends the data sets were divided into $2,4,8,10$, and 16 sections. The sections include 1000, 500, 250, 200, and 125 parts. For the matching of these classes the Munkres algorithm is used [23]. The algorithm for bipartite graphs calculates all possible combinations and the assemble able parts and returns the best matching of the used sections. Due to the fact that the algorithm uses a $\mathrm{N} \times \mathrm{N}$ matrix, only scenarios where the parts have the same number of sections could be used.

\section{Results}

The results show that the length of sections has a high impact on the number of the matched parts. An increasing number of sections leads to an increasing number of assemblies in all 4 scenarios, as shown in Figure 7. The influence of the number of sections is much higher in the scenarios 2 and 4, where opposing trends are simulated.
By the intersection of these two trends in total a higher number of parts could be matched successfully.

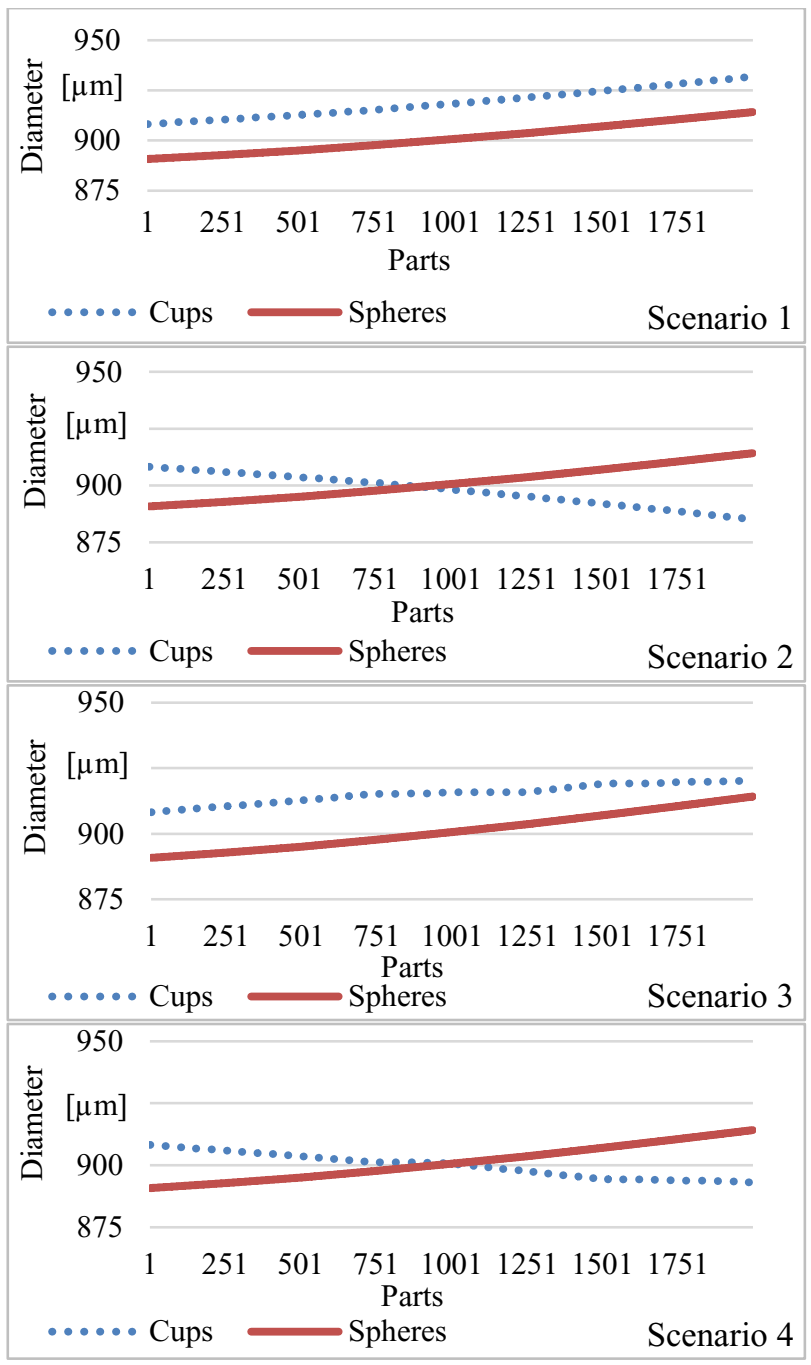

Fig. 6. Simulated trend scenarios. 
In the first and third scenario, where the trends show the same direction, the number of sections has a lower influence on the number of successfully matched parts. The scenarios 1.2, 2.2,3.2, and 4.2, where one trend has a much higher intensity than the other one, the influence of the number of section has also a lower impact on the successful matches.

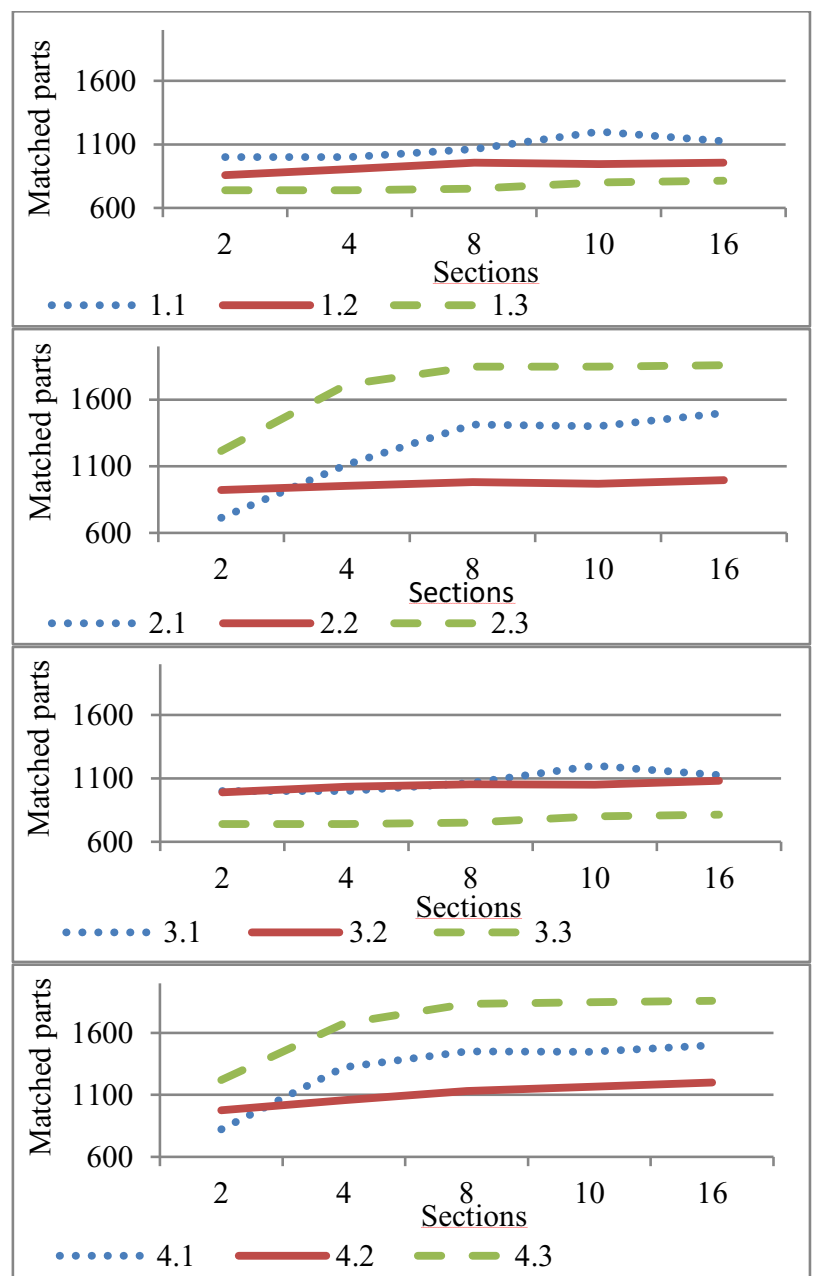

Fig. 7. Successfully matched parts of the sections of the scenarios.

Furthermore, the results indicate that the consideration of the trends influences the output while matching sections. The increases of the output are more significant in the first sections. The number of eight sections is equal with the number of the trends. Therefore, these sections are trend-specific.

Looking at the successfully matched parts at a number of eight sections, it is significantly recognizable that due to the consideration of trends in six scenarios local optima occurs. As listed in Table 2 in the scenarios 1.2, 2.1, 2.2, $3.2,3.3$, and 4.1 the output decreases by the increase of eight to ten sections.

Especially in the scenario 1.2, where both parts show an increasing trend and one of them has a higher intensity, the trends have an influence on the results. Although the number of sections increases further, the found number of matched parts using 8 sections could not be enhanced by the consideration of 10 , or 16 sections.
Table 2. Rise/fall of the output from 8 to 10 sections.

\begin{tabular}{|c|c|}
\hline Scenario & $\begin{array}{l}\text { Rise/fall of the output from } 8 \text { to } \\
10 \text { sections }\end{array}$ \\
\hline 1.1 & $12,89 \%$ \\
\hline 1.2 & $-1,26 \%$ \\
\hline 1.3 & $6,67 \%$ \\
\hline 2.1 & $-0,99 \%$ \\
\hline 2.2 & $-1,32 \%$ \\
\hline 2.3 & $-0,11 \%$ \\
\hline 3.1 & $12,78 \%$ \\
\hline 3.2 & $-0,57 \%$ \\
\hline 3.3 & $6,67 \%$ \\
\hline 4.1 & $-0,28 \%$ \\
\hline 4.2 & $3,01 \%$ \\
\hline 4.3 & $0,71 \%$ \\
\hline
\end{tabular}

Due to the similar intensities of quadratic and linear trends in the third and fourth scenarios, these changes do not have a high impact on the ability of matching these sections.

\section{Summary and Outlook}

The paper introduces an approach for micro mass production that considers conveyance and synchronisation technologies as well as an adapted process design. The design of the parts as well as the synchronisation of these parts interdepends on each other. Therefore, different synchronisation scenarios as well as the synchronisation station for the example of cups and spheres are introduced.

For an additional improvement of the output rates, the tolerances can be softened by building trend-specific sections. For this reason, the influence of trends and trend changes on the number of successfully match able parts is investigated. The results show that especially occurring opposite trends with similar intensities according to the gradient show an increasing number of joinable parts by matching sections. This is caused by the intersection of the two parts. If only one type of linked parts shows a high intensity, appearing intersections are low and for this reason, the effect of building sections is less intensive. In further research it has to be investigated how the number of successfully matched parts of these scenarios could be increased by the adjustment of the nominal value.

The results of the tolerance field widening are implemented in the synchronisation station. Looking at the limited numbers of interruptions for enhancing mass production, the local optima confirm that the trend changes also have an impact in half of the simulated scenarios and that the consideration of the trend changes for the building of sections is an appropriate reference point for the building of sections.

For an assessment of a number of allowed interruptions and synchronisation scenarios, the cycle times of the synchronisation station are implemented in a simulation scenario in future research. Furthermore, the 
influence of real trends on the process times of the synchronisation station and the diameter changes of parts has to be considered. Therefore, parts have to be produced and measured. Due to the fact that varying sizes of trendspecific sections are possible, the matching of flexible section sizes is required.

\section{Acknowledgements}

The authors gratefully acknowledge the financial support by Deutsche Forschungsgemeinschaft (DFG, German Research Foundation) for Subproject C5 "Teileverbunde" within the SFB 747 (Collaborative Research Center) "Mikrokaltumformen - Prozesse, Charakterisierung, Optimierung".

\section{References}

1. F. Vollertsen, D. Biermann, H.N. Hansen, I.S. Jawahir, K. Kuzman, Size effects in manufacturing of metallic components, CIRP Annals - Manufacturing Technology, 58/2, 566-587 (2009)

2. K. Tracht, F. Weikert, Handling of Micro Parts, Micro Metal Forming, Springer-Verlag Berlin Heidelberg 331-342 (2013)

3. H.N. Hansen, K. Carneiro, H. Haitjema, L. De Chiffre, Dimensional Micro and Nano Metrology, Annals of the CIRP, 55/2, 721-743 (2006)

4. P. Groche, R. Schneider, Method for Optimization of Forming Presses for the Manufacturing of Micro Parts, Annals of the CIRP, 53/1, 281-284 (2004)

5. K. Tracht, C. Schenck, F. Weikert, B. Kuhfuss, Förderung mikrokaltumgeformter Teile im Verbund (Conveyance of micro-cold-formed parts in a linkage - Overcoming of size effects by connecting microcold-formed parts), wt werkstattstechnik online, 100/11/12:864-868 (2010) - in German

6. M.S. Kumar, S.M. Kannan, Optimum manufacturing tolerance to selective assembly technique for different assembly specifications by using genetic algorithm. Int J Adv Manuf Technol, 32, 591-598 (2007)

7. G. Lanza, B. Haefner, A. Kraemer, Optimization of selective assembly and adaptive manufacturing by means of cyber-physical system based matching, CIRP Annals - Manufacturing Technology, 64/1, 399402 (2015)

8. M. Colledani, D. Ebrahimi, T. Tolio, Integrated Quality and Production Logistics Modelling for the Design of Selective and Adaptive Assembly Systems, CIRP Annals - Manufacturing Technology, 62/1, 58 (2014)

9. M.V. Raj, S.S. Sankar, S.G. Ponnambalam, Minimizing Clearance Variations and Surplus Parts in Multiple Characteristic Radial Assembly Through Batch Selective Assembly. Int J Adv Manuf Technol, 57, 1199-1222 (2011)

10. A.-K. Onken, P. Rückert, C. Perl, K. Tracht, Joining linked micro parts by tolerance field widening and synchronization, Tagungsband des 2. Kongresses
Montage Handhabung Industrieroboter, SpringerVerlag Berlin Heidelberg, 1-8 (2017)

11. H.C. Meng, K.C. Ludema, Wear models and predictive equations : their form and content, Wear, 181-183, 443-457 (1995)

12. S.M. Hsu, M.C. Shen, A.W. Ruff, Wear prediction for metals, Tribology International, 30/5, 377-383 (1997)

13. R.B. Abernethy, The New Weibull Handbook, 5, (2010)

14. N.P. Belfiore, F. Ianniello, D. Stocchi, F. Casadei, D. Bazzoni, A. Finzi, S. Carrara, J.R. González, J.M. Llanos, I. Heikkila, F. Peñalba, X. Gómez, A hybrid approach to the development of a multilayer neural network for wear and fatigue prediction in metal forming, Tribology International, 40 1705-1717 (2007)

15. D. Yilkiran, D. Wulff, A. Almohallami, F. Özkaya, A. Bouguecha, S.Hübner, K. Möhwald, H.J. Maier, B.-A. Behrens, Wear behaviour of thermally oxidised tool surfaces as low-friction separation layers for dry sheet metal forming, Wear, 376-377, 1789-1803 (2017)

16. Z.Q. Liu, X. Ai, H. Zhang, Z.T. Wang, Y. Wan, Wear patterns and mechanisms of cutting tools in highspeed face milling, Journal of Materials Processing Technology, 129, 222-226 (2002)

17. A. Ber, S. Kaldor, The First Seconds of Cutting, Wear Behaviour, Annals of the CIRP, 31/1, 13-17 (1982)

18. C. Boher, D. Attaf, L. Penazzi, C. Levaillant, Wear behaviour on the radius portion of a die in deepdrawing: Identification, localisation and evolution of the surface damage, Wear, 259, 1097-1108 (2005)

19. H. Flosky, F. Vollertsen, Wear behaviour on a combinded micro blanking and deep drawing process, CIRP Annals- Manufacturing Technology, 63, 281-284, 2014

20. F. Weikert, J. Weyhausen, K. Tracht, Statistical Study for Micro Forming Technologies Used for Linked Parts Production, $5^{\text {th }}$ International Conference on Changeable, Agile, Reconfigurable and Virtual Production, Springer International Publishing Switzerland,41-46 (2016)

21. P. Wilhelmi, C. Schenck, B. Kuhfuss, Diameter adaptive guides for wire based linked micro parts. Proceedings of the 4M/IWMF Conference, Lyngby, Denmark, 13.09.-15.09.2016

22. P. Wilhelmi, C. Schenck, B. Kuhfuss, High Speed Referencing System for Linked Micro Parts, Proceedings of the ICOMM - International Conference on Micro Manufacturing 2016. Orange County, California (USA), March 29.03.-31.03.2016.

23. J. Munkres, Algorithms for the Assignment and Transportation Problems, Journal of the Society for Industrial and Applied Mathematics, 5/1, 32-38 (1957) 